\title{
Signal Processing Technique for Identifying Pacifier Artifacts in Pediatric Sleep Lab Airflow Data
}

\author{
Padmini Selvaganesan $^{1 *}$, Michala Dauterman ${ }^{1}$, Ajay Mahajan $^{2}$ and Jyoti Krishna ${ }^{3}$ \\ ${ }^{1}$ Department of Biomedical Engineering, The University of Akron, Ohio, USA \\ ${ }^{2}$ Department of Mechanical and Biomedical Engineering, The University of Akron, Ohio, USA \\ ${ }^{3}$ Pediatric Sleep Medicine, Akron Children's Hospital, Ohio, USA
}

*Corresponding author: Padmini Selvaganesan, Department of Biomedical Engineering, The University of Akron, 264 Wolf Ledges, 211A, Akron, OH 44325, USA, Tel: +1-3135592302, E-mail: ps120@zips.uakron.edu

\begin{abstract}
For diagnosing sleep apnea, patients are required to stay overnight in a sleep lab, and various physiological signals are recorded using different sensors. The data collected during the study is often prone to artifacts due to various reasons and one such artifact in younger patients is due to the use of pacifiers which corrupts the signal from the sensors. One of the sensor signals which is corrupted frequently is the airflow signal. This airflow signal is obtained using a thermistor that is placed just below the nostrils. Thermistor readings are used to determine the airflow and breathing pattern in the patients based on the difference in temperature readings of the air that is drawn in and then breathed out. The objective of this study is to develop a wavelet based signal processing technique to identify and remove such artifacts from the thermistor data. Wavelet technique is first developed and tested on a simulated waveform to remove artifacts, and then validated on the actual waveform obtained from a patient. The technique shows satisfactory output in removal of artifacts and in reconstruction of the actual signal. It must be noted here that the removal of the artifact may not provide information on the occurrence of a sleep apnea episode by that sensor, but directs more attention to other sensors to see if there was an episode at that time. In addition, the identification and removal of the artifact is the first stage towards an automatic software-based scoring system in the future.
\end{abstract}

\section{Keywords}

Wavelets, Sleep apnea, Air flow measurements, Artifacts

\section{Abbreviations}

OSA: Obstructive Sleep Apnea; CWT: Continuous Wavelet Transformation; STFT: Short-Time Fourier Transform

\section{Introduction}

Pediatric sleep study scoring involves a lengthy and laborious process highlighting the need for error free data. These studies normally take place in hospital sleep labs and require the patient to relocate to the hospital for the night, get attached to hospital lab sensors, be monitored while sleeping throughout the night and leave sometime in the next morning. While the actual sleep study for sleep apnea usually only takes one night to run, the preparation for the study must be started well in advance. It may take several months from the time a doctor orders an in-lab sleep study, to the time the patient gets an appointment. This is due to the limited number of sleep labs relative to the demand. Following the actual study, it may additionally take several days to score and interpret the raw data and confirm a diagnosis of, say, Obstructive Sleep Apnea (OSA). The presence of artifacts, or a malfunctioning sensor, makes scoring for OSA even more difficult. The compromised data may lead to the patient being misdiagnosed or needing to go through the months-long process again to obtain better data. Because of the lengthy process involved in sleep lab studies, it is crucial to maximize acquisition of uncorrupted sleep data at the outset.

In sleep studies involving children, the ability to gain a good set of data is more challenging than it is with adults. Children tend to move more and inadvertently dislodge the sensors causing corrupted data and misanalysis. It is common to see airflow signal artifact in

Citation: Selvaganesan P, Dauterman M, Mahajan A, Krishna J (2019) Signal Processing Technique for Identifying Pacifier Artifacts in Pediatric Sleep Lab Airflow Data. Int J Pediatr Res 5:055. doi. org/10.23937/2469-5769/1510055

Accepted: September 14, 2019: Published: September 16, 2019

Copyright: (C) 2019 Selvaganesan P, et al. This is an open-access article distributed under the terms of the Creative Commons Attribution License, which permits unrestricted use, distribution, and reproduction in any medium, provided the original author and source are credited. 
the subset of young children that use a pacifier during sleep. The child's act of sucking on the pacifier causes movement artifact of specifically the oro-nasal sensor used to monitor airflow during respirations. This can result in inaccurate scoring of sleep apnea in this segment of children. There are numerous studies in the literature that have reported on the pros and cons of pacifier sucking among children, the development of sleeping and breathing patterns in children, and the development of non-nutritive sucking in children [1-4]. But the impact of pacifier use on a sensor and its data is not discussed widely.

\section{Problem Definition}

With the current technology available in sleep labs, the use of a pacifier often hinders the data collection by airflow sensors. Yet it is also true that the pacifier-dependent child cannot quietly fall asleep without their pacifier, so they cry and become restless. This action too can cause the sensors to be bumped out of place and then must be reset.

While the pacifier does calm the children down so they can fall asleep, it also affects the sensors, specifically the oro-nasal sensor, because of the to-and-fro motion created as the child sucks. A thermistor is used as oro-nasal sensor which is placed above the mouth or under the nose to determine the airflow while breathing and to calculate the respiratory rate. The oro-nasal sensor used has three prongs on it. One lower prong rests on the upper lip of the child and two upper prongs rest at the nares. The sucking motion causes movement of the pacifier and the pacifier causes movement to the lower prong on the oro-nasal sensor. The movement of the lower prong is what creates an artifact in the sleep data and disrupts the study Figure 1.

The movement of the pacifier can also dislodge the oro-nasal sensor so it can no longer properly read the data, and thus this sensor must be repositioned to gain good non-compromised data. In these cases, the sleep technician will attempt to reposition the sensor after the child is asleep but this action may result in waking up the child and the process must start all over again.

The main objective of this study was to develop a signal processing technique to identify and remove such artifacts created by the pacifier in sensor readings. It was hypothesized that signal processing techniques can be used to remove the artifacts in the frequency domain and then reconstruct the original signal knowing that one may have lost the ability to identify a sleep apnea episode using that sensor. But these timestamps can still be flagged, and more emphasis can be given to other sensors such as pulse-oximeter and/or effort belt to determine the oxygen saturation and breathing effort at these times. In addition, cleaning the signals of artifacts is still the first stage to eventually advancing towards an automatic scoring system of sleep lab data.

\section{Methods}

\section{Design -Artifact Removal.}

Signal Processing Technique Development on Simulated Data.

A signal processing technique was developed to remove the artifacts from thermistor sensor readings that were created by pacifier use. The typical frequency of breaths is approximately 30 breaths/minute in children under the age of two years, while the artifacts have typically higher frequency than the breathing frequency. Normal breath rates for children are shown below Table 1 [5].

This paper is focused on the output of the thermistor (oro-nasal) sensor. The typical airflow waveform from the sensor is shown in Figure 2. These are essentially voltage readings based on temperature change of the air that is expired from the nose or mouth at a higher temperature than the ambient temperature of the room.

For young children that use pacifiers, there are artifacts that are picked up by the sensor and the data-acquisition system. These artifacts are at a higher

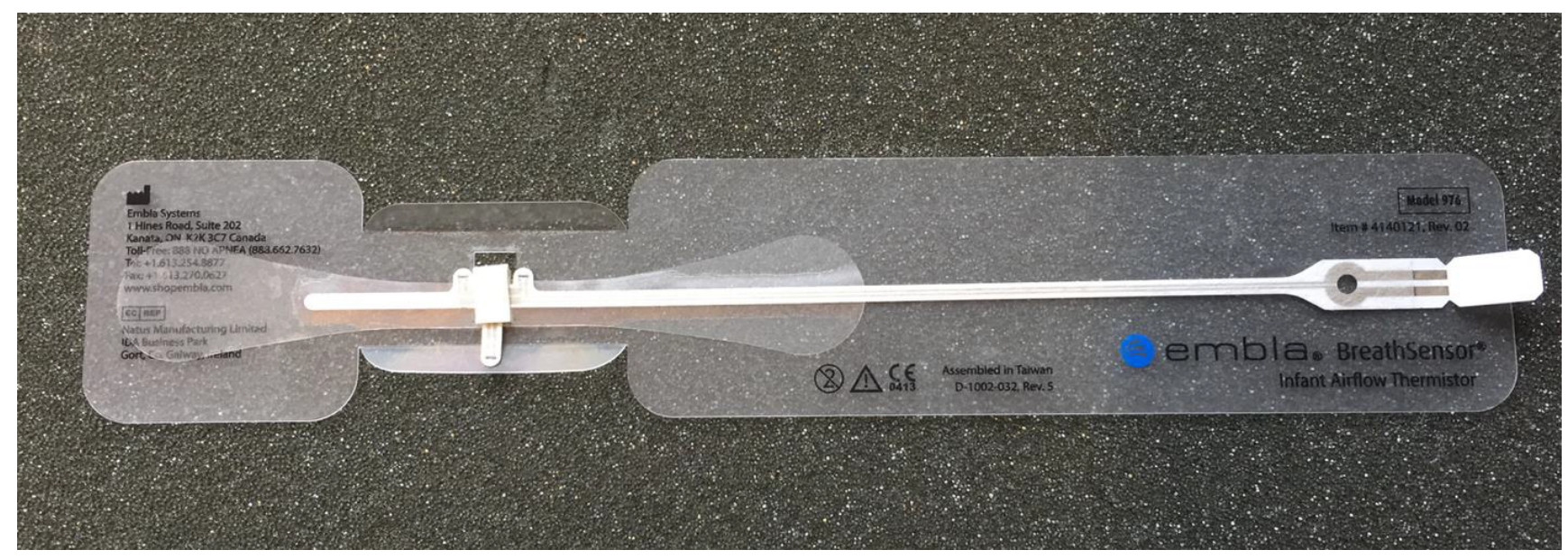

Figure 1: Thermistor - airflow sensor. 
Table 1: Respiratory rate.

\begin{tabular}{|l|l|}
\hline Age Range (Years) & Breath per minute \\
\hline $0-1$ & $30-60$ \\
\hline $1-2$ & $30-60$ \\
\hline $2-3$ & $24-40$ \\
\hline $3-4$ & $24-40$ \\
\hline $4-5$ & $22-34$ \\
\hline $5-6$ & $22-34$ \\
\hline $6-12$ & $18-30$ \\
\hline $12-13$ & $18-30$ \\
\hline $13-18$ & $12-16$ \\
\hline
\end{tabular}

frequency than natural breathing frequency of the child, and mask the actual useful signal. A sample of this artifact is indicated by the arrow in Figure 3 .

A continuous wavelet transformation (CWT) technique was developed to remove the pacifier artifact from the thermistor readings. A wavelet is a wave like oscillation with an amplitude that begins at zero, increases, and then decreases back to zero. It can be visualized as a brief oscillation like one recorded by a heart monitor. Using a "reverse, shift, multiply and integrate" technique called convolution, wavelets can be combined with known portions of a damaged signal to

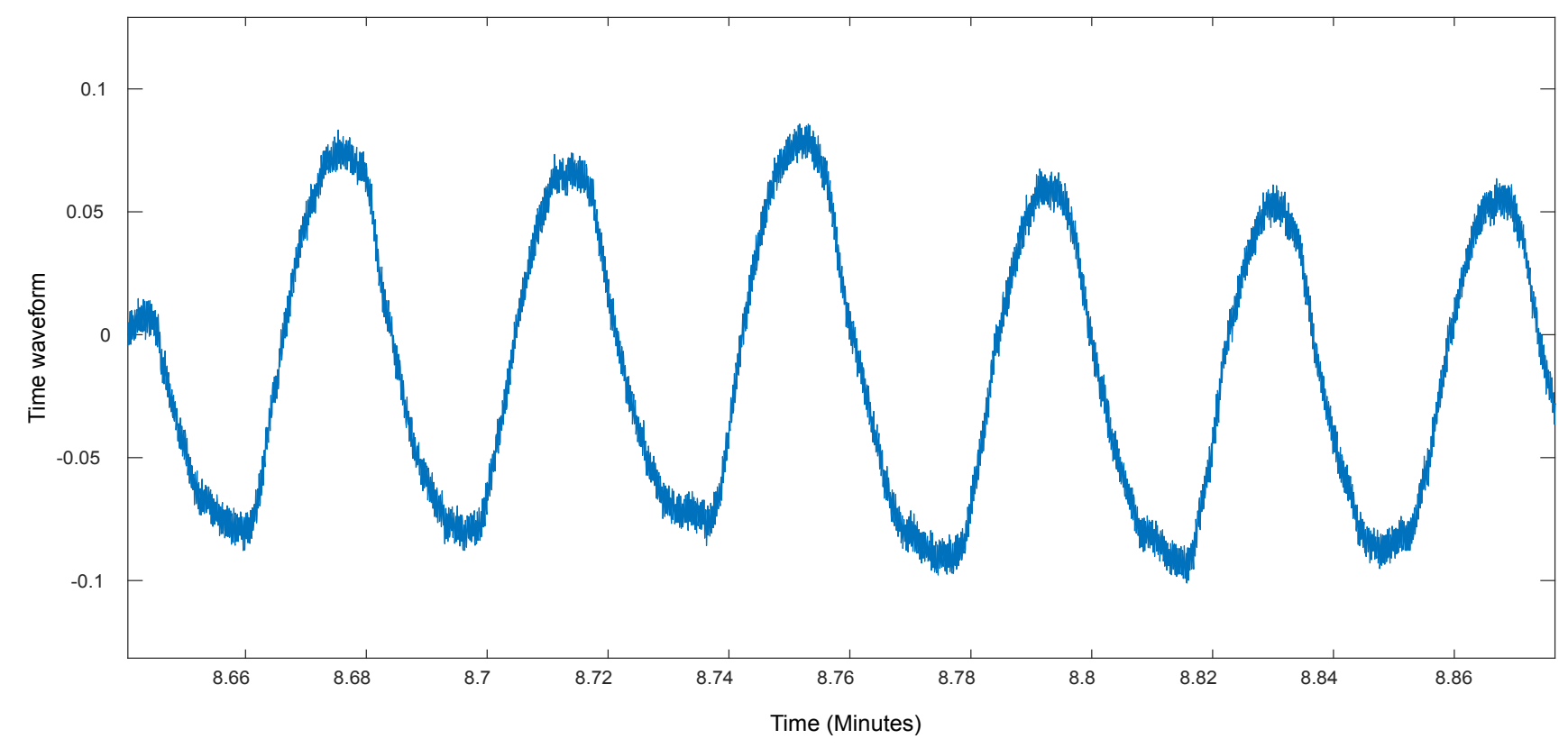

Figure 2: Thermistor data from patient.

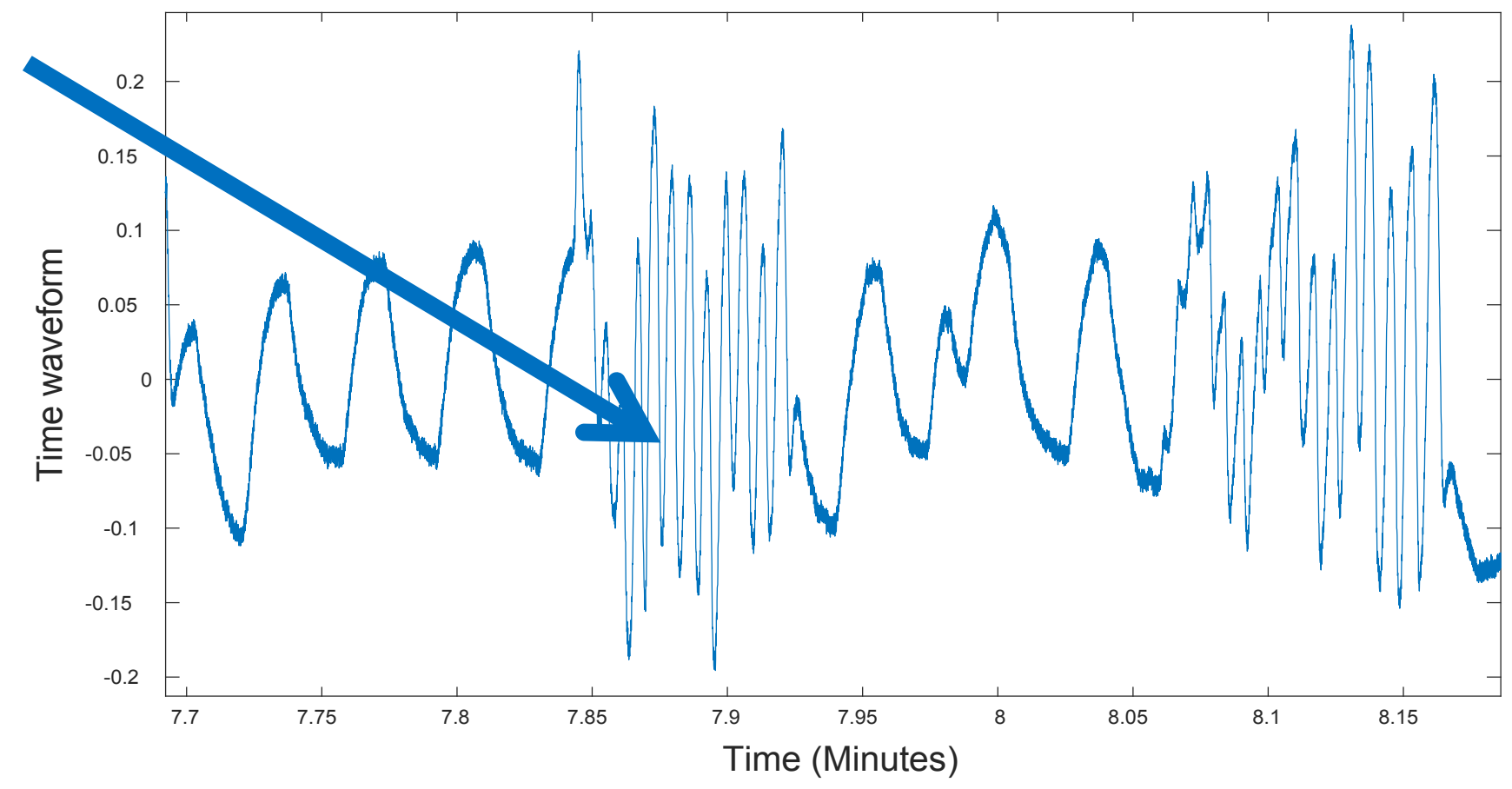

Figure 3: Artifact due to pacifier in thermistor data. 


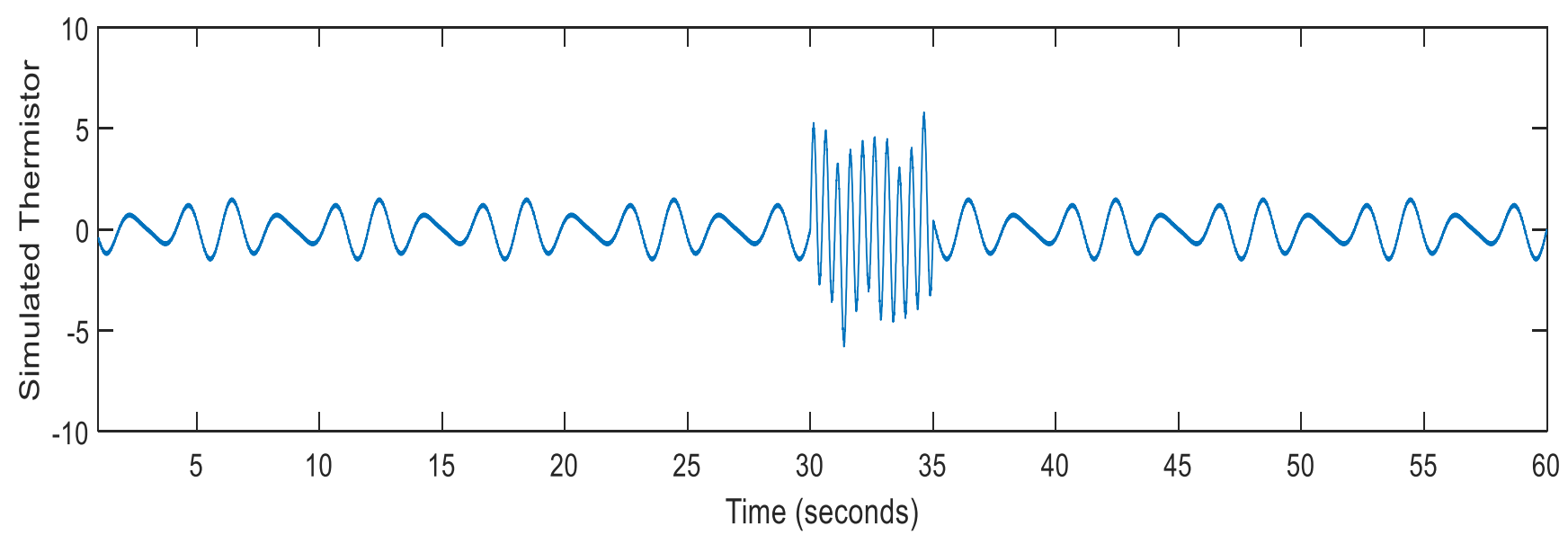

Figure 4: Simulated thermistor data with pacifier artifact.

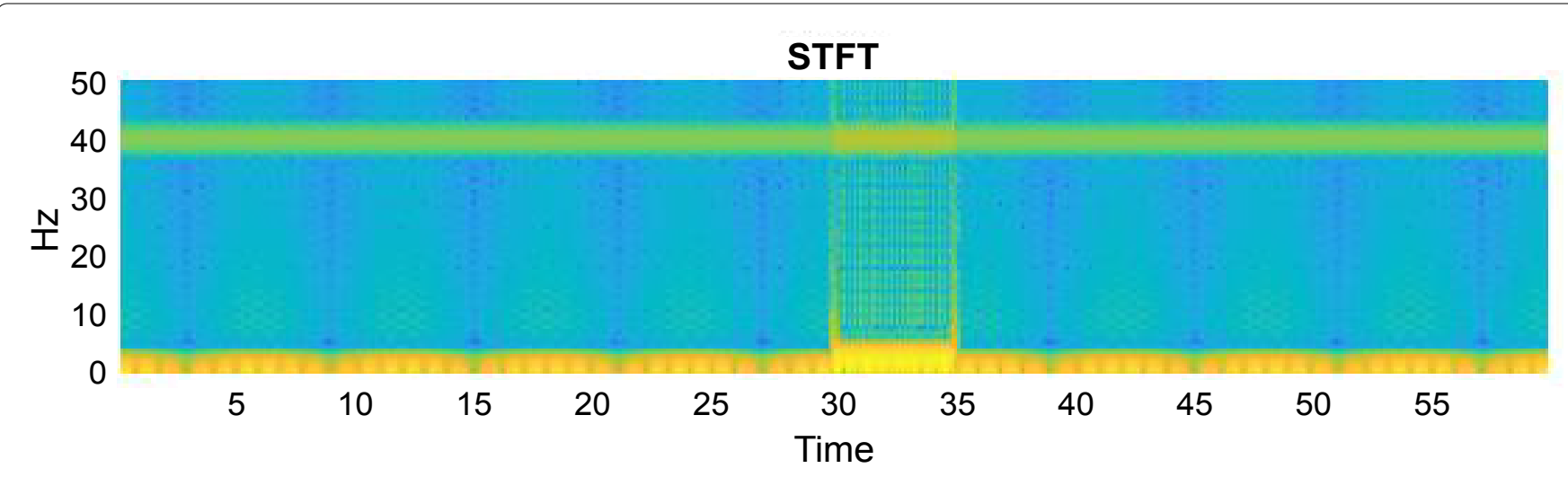

Figure 5: Short time fourier transform of the simulated data.

extract information from the unknown portions [6].

In continuous wavelet transforms, a given signal of finite energy is projected on a continuous family of frequency bands. For instance, the signal may be represented on every frequency band of the form $[f, 2 f]$ for all positive frequencies $f>0$. Then, the original signal can be reconstructed by a suitable integration over all the resulting frequency components [7].

Applying this theory to the thermistor artifact that occurs during pacifier use, initially a simulated signal corresponding to the 30 breaths/minute $(0.5 \mathrm{~Hz})$ was created (sampling frequency $100 \mathrm{~Hz}$ ). An artifact lasting for 5 seconds is induced between 30-35 seconds with a $5 \times$ frequency and was superimposed on the simulated signal. This is shown in Figure 4 that is given below.

Next, a short-term Fourier transform (STFT) of the combined simulated signal was generated. An STFT is typically used to determine the sinusoidal frequency and phase content of local sections of a signal as it changes over time. In practice, the procedure for computing STFTs is to divide a longer time signal into shorter segments of equal length and then compute the Fourier transform separately on each shorter segment. This reveals the Fourier spectrum on each shorter segment. Usually the changing spectra is then plotted as a function of time [8]. The resulting STFT for the simulated thermistor data is shown in Figure 5 given below.

Following the STFT, a Morlet Wavelet was used to identify the artifact. The Morlet wavelet composed of a complex exponential multiplied by a Gaussian window. The Morlet wavelet transform method offers an intuitive bridge between frequency and time information which can clarify interpretation of complex spectra obtained with STFT. The Morlet wavelet transform, however, is not intended as a replacement for STFT, but rather a supplement that allows qualitative access to time related changes and takes advantage of the multiple dimensions available in a free induction decay analysis [9]. The resulting Morlet wavelet identifying the thermistor artifact caused by a pacifier is shown in Figure 6 given next.

Having the Morlet wavelet, a CWT was done to find the frequencies of the artifact and their locations. This is shown in Figure 7 given below. Finally, a filter was used to block off the artifact frequencies and an inverse CWT was used to recreate the time-wave back. This is shown in Figure 8.

\section{Results}

The artifact frequencies were between 1 and $6 \mathrm{~Hz}$. 


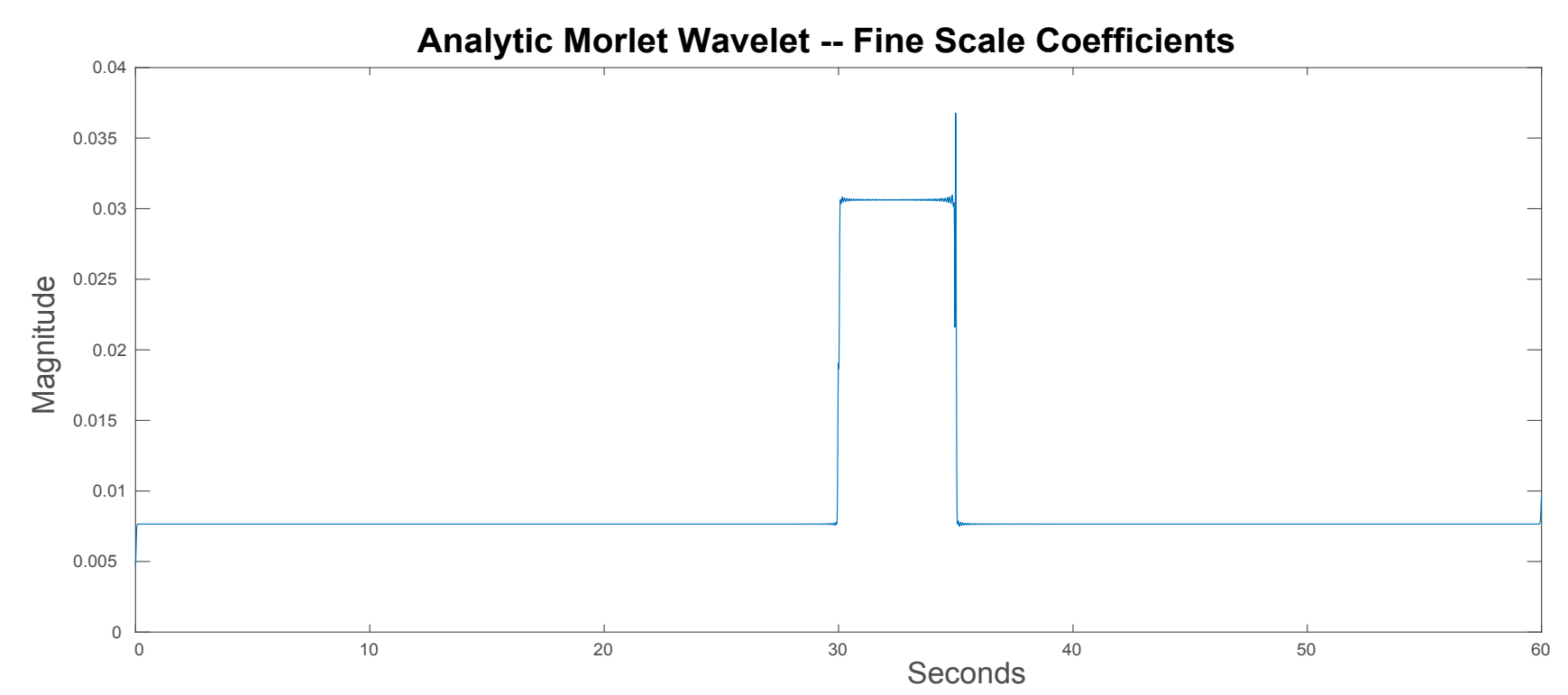

Figure 6: Morlet wavelet fine scale coefficients.

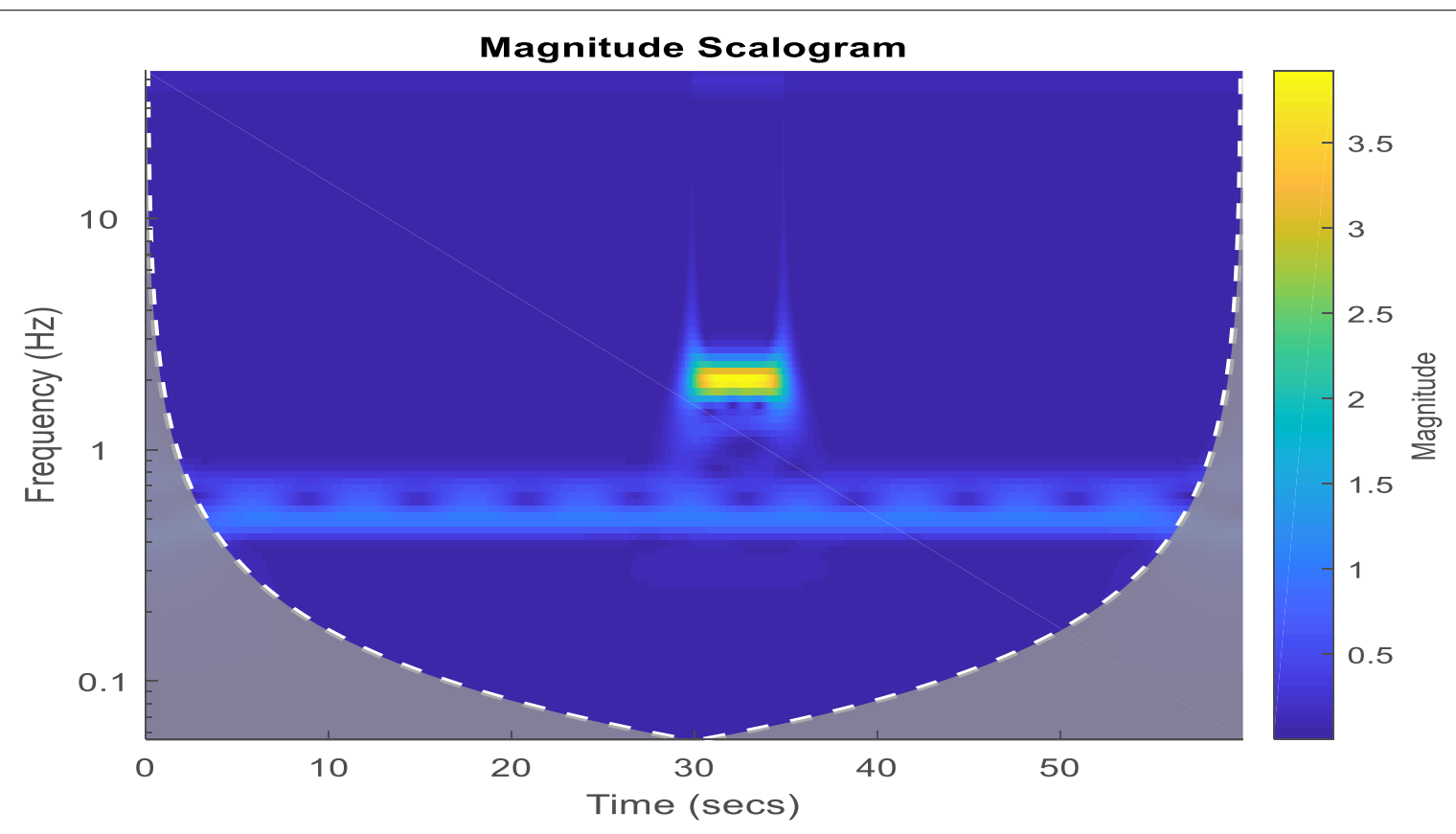

Figure 7: Artifact identified.

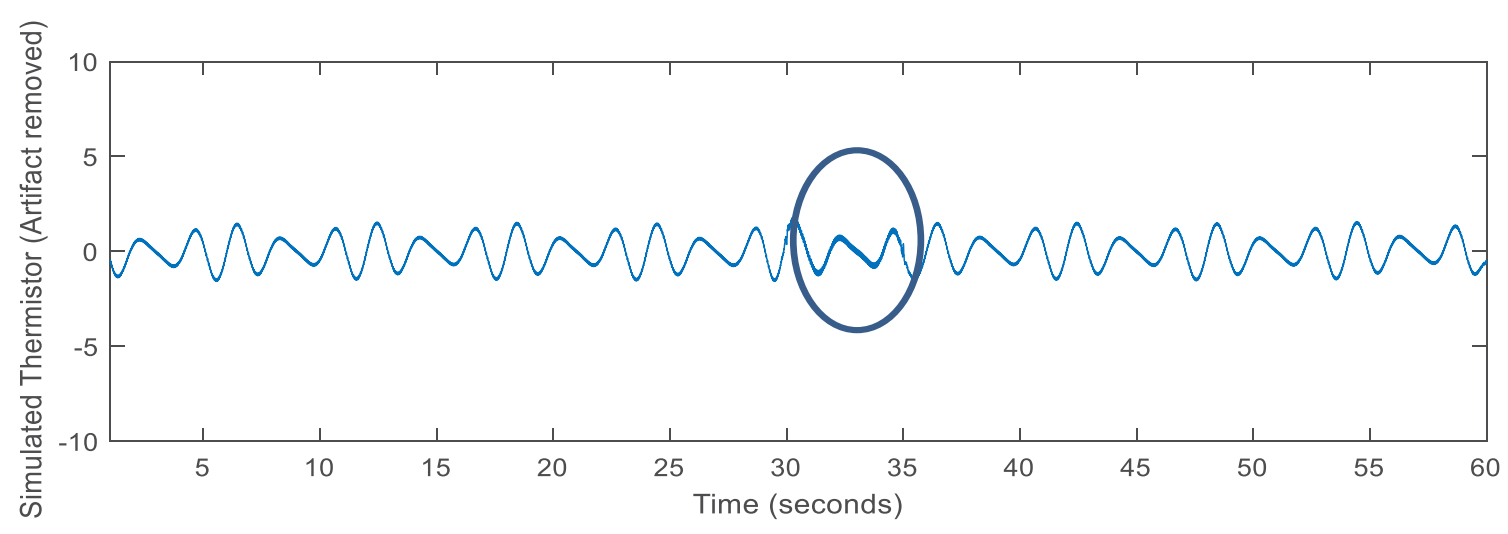

Figure 8: Artifact free simulated thermistor data.

They lasted for 5 seconds between 30-35 seconds. simulated thermistor reading. In fact, the entire signal This information was used to remove artifacts from a could be cleaned since the useful signal frequency is 


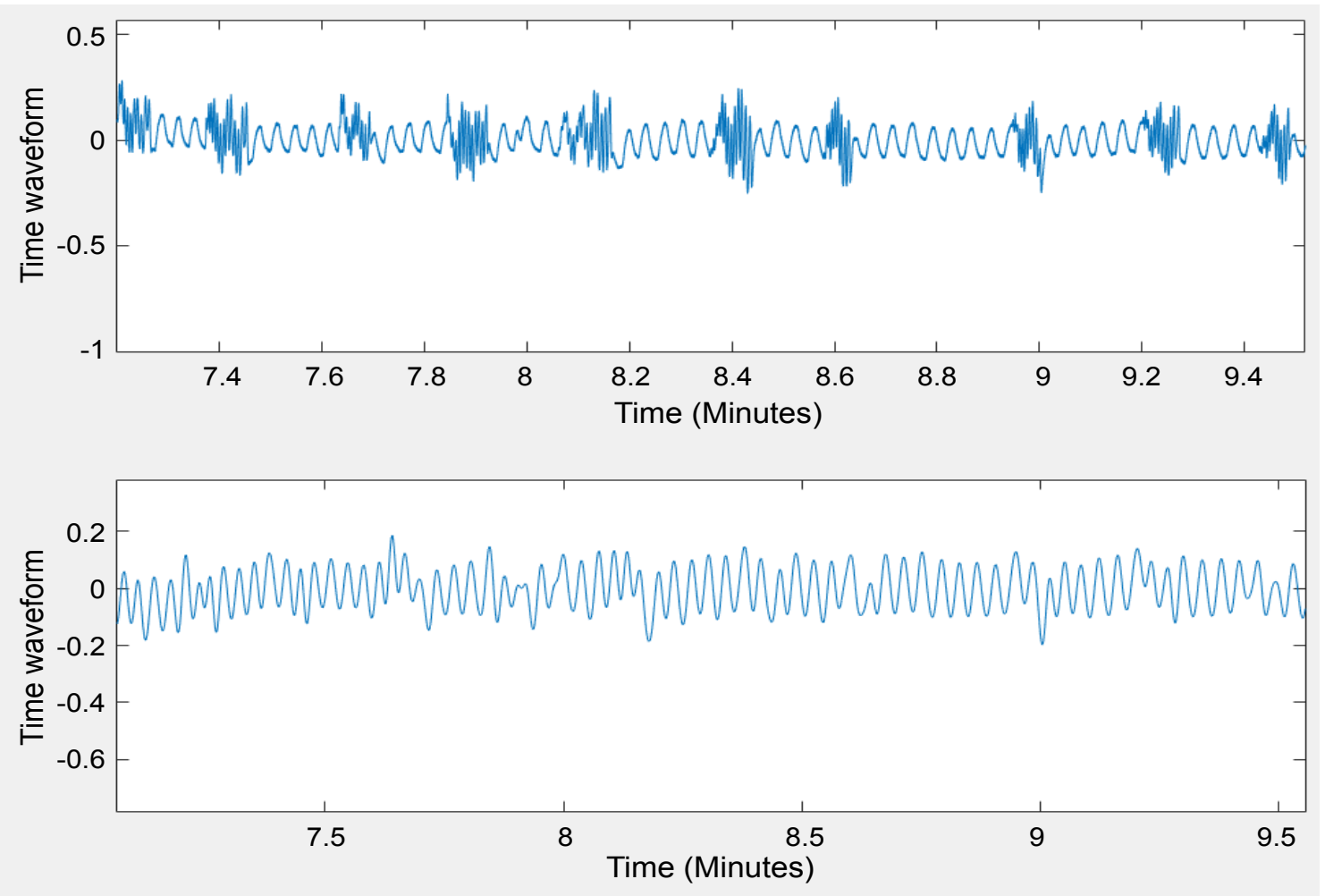

Figure 9: Patient airflow data.

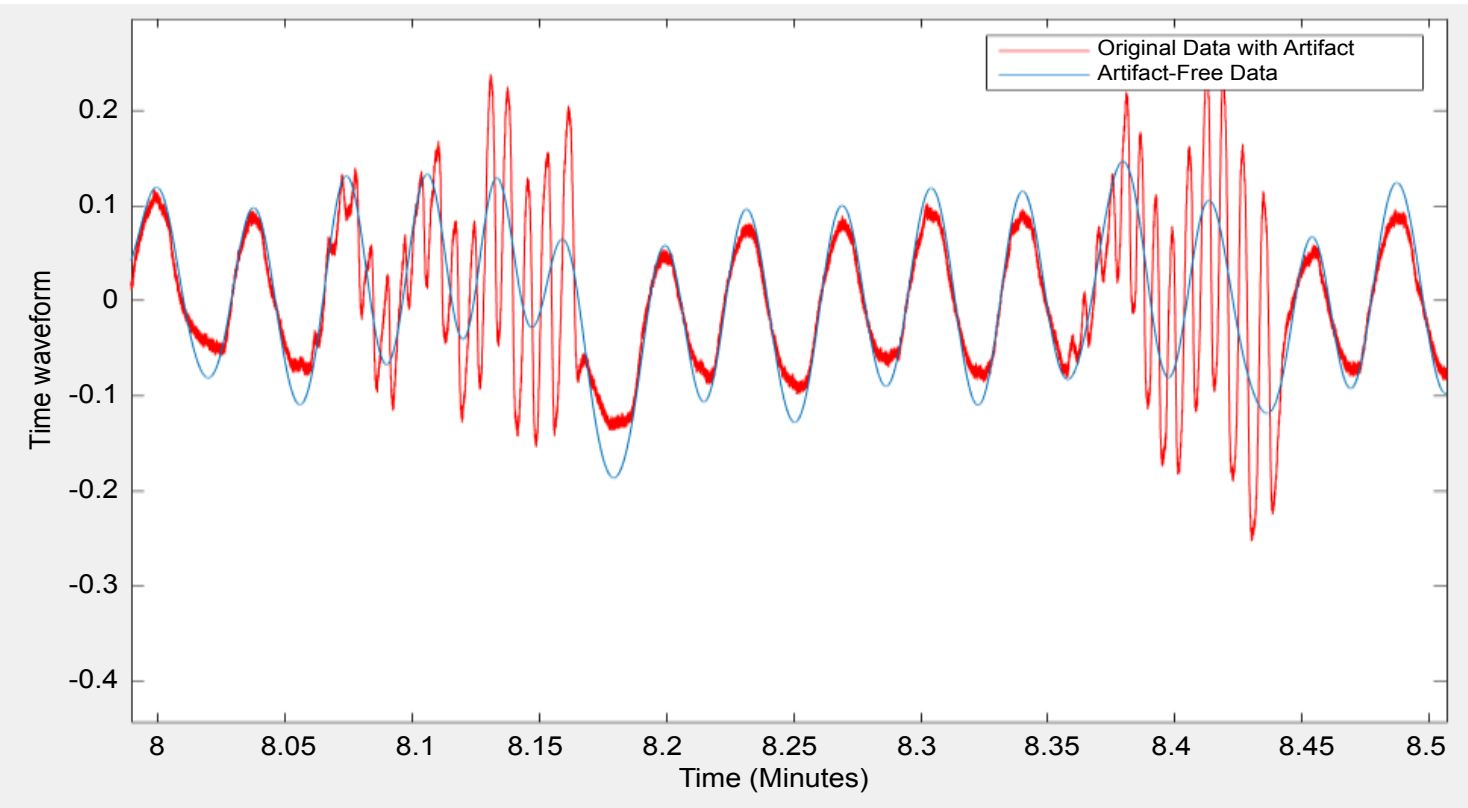

Figure 10: Original data with artifact vs. artifact free data.

below $0.5-1 \mathrm{~Hz}$.

\section{Signal Processing Technique on Actual Data}

Having successfully developed a signal processing technique to remove a simulated artifact from the simulated data, it was hypothesized that the technique would then work on actual recorded data with pacifier artifacts. Actual sleep lab data was then put through the signal processing technique developed to see if the developed technique could accurately remove the artifacts. The top waveform in Figure 9 shows the airflow data obtained from a few months old patient using a thermistor for a short duration of time. The high frequency waves are due to the intermittent usage of pacifier. The second one is artifact free waveform obtained after going through the wavelet transformation.

Figure 10 provides a clear picture of the original data against the artifact free data. The hypothesis was proven correct, in that the technique worked very well on actual sleep lab data, and was successful in identifying and removing pacifier created artifacts. 


\section{Conclusion}

This paper demonstrates that the wavelet technique developed for the study can not only be used to clean up the artifacts in the obtained data but also to obtain an estimate of the actual signal buried under the artifact. One must still be careful to study other sensor readings during these times. The work proposed in this study can help in better visualization of the data which results in improved analysis by the technician and the physician. In addition this work paves the way for automatic scoring of sleep lab data in the future.

\section{Acknowledgement}

The authors would like to acknowledge Akron Children's Hospital for supporting and funding this study.

\section{Conflict of Interest}

There is no conflict of interest to disclose.

\section{Source of Support}

Akron Children's Hospital.

\section{References}

1. Adair SM (2003) Pacifier use in children: A review of recent literature. Pediatr Dent 25: 449-458.

2. Lau Chantal (2015) Development of suck and swallow mechanism in infants. Ann Nutr Metab 66: 7-14.

3. MacLean JE, Fitzgerald DA, Waters KA (2015) Developmental changes in sleep and breathing across infancy and childhood. Paediatr Respir Rev 16: 276-284.

4. Oder AL, David L Stalling, Steven M Barlow (2013) Shortterm effects of pacifier texture on nns in neurotypical infants. International Journal of Pediatrics.

5. Fleming S, Thompson M, Stevens R, Heneghan C, Plüddemann $A$, et al. (2011) Normal ranges of heart rate and respiratory rate in children from birth to 18 years of age: A systematic review of observational studies. Lancet 377 : 1011-1018.

6. Ricker Norman (1953) Wavelet contraction, wavelet expansion and the control of seismic resolution. Geophysics 18: 769-792.

7. Larson David R (2007) Unitary systems and wavelet sets.

8. Sejdic E, Igor Djurovi'c, Jin Jiang (2009) Time-frequency feature representation using energy concentration: An overview of recent advances. Digital Signal Processing 19: 154-162.

9. Frederick S, Alexander L, Hunter CS, Nick P, Brian R (2001) Definition of the neurochemical patterns of human head injury in ${ }^{1} \mathrm{H}$ MRS using wavelet analysis. Proc Intl Soc Mag Reson Med. 